\title{
CLOSED-LOOP RIGID MODEL IDENTIFICATION OF AN INDUSTRIAL ROBOT
}

\author{
Basilio Bona ${ }^{* 1}$ Aldo Curatella* \\ * Dipartimento di Automatica e Informatica \\ Politecnico di Torino \\ Corso Duca degli Abruzzi 24, 10129 Torino, Italy \\ basilio.bona@polito.it
}

\begin{abstract}
The identification process aimed at estimating the model parameters of a COMAU Smart S2 industrial robot for controller design purposes is presented. The principal challenges include the existence of a controller-in-the-loop and the absence of joint sensors for acceleration and velocity measurements. A method valid in this context has been applied, and suitable trajectories were generated to avoid the excitation of the unmodelled dynamics. This method was applied to an industrial robot, its parameters estimated and used for the design of a model-based controller. Copyright (C)2005 IFAC
\end{abstract}

Keywords: model identification, base parameters estimation, closed-loop identification, robot dynamics.

\section{INTRODUCTION}

To improve robot geometric performances it is often necessary to design advanced model-based controllers that require an accurate estimate of the model parameters. Since the robot producers very rarely provide this information, users need to follow a reliable procedure allowing to recover a model valid in the frequency range of interest.

The dynamic model of a robot depends on such parameters as links inertia, mass and center of mass, but only a subset of them, called Base $P a$ rameters (Gautier and Khalil, 1990; Gautier and Khalil, 1988; Mayeda et al., 1990; Pham and Gautier, 1991; Kozlowzki, 1998), must be estimated to avoid a rapid rise in computational complexity when the number of degrees-of-freedom (DoF) grows.

Many identification methods exist for system operating in open loop (Ljung, 1987; Forssell and Ljung, 1999; Forssell and Ljung, 2000; Sun et

\footnotetext{
1 This work was partially supported by MIUR 2002 PRIN "MATRICS" project.
}

al., 2000; Welsh and Goodwin, 2002), but several of them fail when the system is under closed-loop action, as in the robot case, where it cannot operate without an active controller, for safety reasons. One of the methods for closed loop system identification (Ljung, 1987), (Welsh and Goodwin, 2002) shall be used instead; in this paper the Projection Method (PM) was chosen since it does not rely on any hypothesis about the controller structure.

Velocity and acceleration measurements are necessary in the identification process, but often the relevant sensors are not present for cost reasons. To avoid numerical derivation, a Finite Fourier series was used to generate reference trajectories; this allows also to average the data, improve the signal-to-noise ratio, compute statistical characteristic of noise measurements, and define the bandwidth of the exciting trajectory.

Section 2 introduces the robot model (assumed to be rigid) and its parameters, and reviews possible identification methods, while in Section 3 the results obtained implementing the PM method on a real COMAU Smart-3 S2 industrial robot will be 
presented and discussed. Conclusions are drawn in Section 4.

\section{THE ROBOT MODEL}

The assumed model is based on open-chain $n$ links physical model, with the following additional hypothesis: $(i)$ rigid links and joints are assumed; (ii) joint gears are ideal, i.e. $100 \%$ efficient, without dead bands, etc.; ( $i i i$ ) friction is modelled as the sum of viscous and Coulomb friction only, i.e. no stiction is considered.

Lagrange equations are applied for each link $i=$ 1, $\cdots, n$, (Bona, 2002; Kozlowzki, 1998)

$$
\frac{d}{d t}\left(\frac{\partial \mathcal{L}}{\partial \dot{q}_{i}}\right)-\frac{\partial \mathcal{L}}{\partial q_{i}}=\tau_{i}-f_{i}^{f}
$$

where $n$ is the number of joints, $\mathcal{L}$ is the Lagrange Energy Function

$$
\mathcal{L}(\boldsymbol{q}, \dot{\boldsymbol{q}})=\mathcal{C}(\boldsymbol{q}, \dot{\boldsymbol{q}})-\mathcal{P}(\boldsymbol{q})
$$

where $\mathcal{C}$ and $\mathcal{P}$, are respectively, the kinetic coenergy and the potential energy, $\boldsymbol{q} \in \mathbb{R}^{n}$ is the joint position vector, $\dot{\boldsymbol{q}} \in \mathbb{R}^{n}$ the joint velocity vector, $q_{i}$ and $\dot{q}_{i}$ the $i$-th joint position and velocity respectively, while $f_{i}^{f}$ is the friction force acting on the $i$-th joint, modelled as:

$$
f_{i}^{f}\left(\dot{q}_{i}\right)= \begin{cases}\beta_{i}^{v+} \dot{q}_{i}+\beta_{i}^{c+} & \dot{q}_{i}>0 \\ 0 & \dot{q}_{i}=0 \\ \beta_{i}^{v-} \dot{q}_{i}+\beta_{i}^{c-} & \dot{q}_{i}<0\end{cases}
$$

The link parameters to be estimated are the inertial parameters (mass $m_{i}$, inertia tensor $\boldsymbol{\Gamma}_{i}$ and center of mass $\boldsymbol{c}_{i}$ expressed in local coordinate frames assigned to individual axes), and the friction parameters $\left(\beta_{i}^{v+}, \beta_{i}^{v-}, \beta_{i}^{c+}, \beta_{i}^{c-}\right)$.

\subsection{Base Parameters}

Ten inertial parameters must be estimated for every link and, numerical complexity of the procedure increases with the degrees-of-freedom $n$. Fortunately, only the parameters that influence the dynamic behaviour, i.e. the Base Parameters, are to be estimated (Gautier and Khalil, 1988; Pham and Gautier, 1991). Assuming that the inertial parameters of link $i$ are given by

$$
\begin{array}{r}
\boldsymbol{X}^{i}=\left[\Gamma_{i}^{x x}, \Gamma_{i}^{x y}, \Gamma_{i}^{x z}, \Gamma_{i}^{y y}, \Gamma_{i}^{y z}, \Gamma_{i}^{z z}\right. \\
\left.m x_{i}, m y_{i}, m z_{i}, m_{i}\right]^{\top}
\end{array}
$$

where $\Gamma_{i}^{x x}, \ldots, \Gamma_{i}^{z z}$ are the elements of the inertia tensor $\boldsymbol{\Gamma}_{i}$, and $m_{i}$ is the mass of link $i$, we can write $\mathcal{C}$ an $\mathcal{P}$ as:

$$
\mathcal{C}=\sum_{j=1}^{10 n} \frac{\partial \mathcal{C}}{\partial X_{j}} X_{j}=\sum_{j=1}^{10 n} D \mathcal{C}_{j} X_{j}
$$

$$
\begin{array}{r}
\mathcal{P}=\sum_{j=1}^{10 n} \frac{\partial \mathcal{P}}{\partial X_{j}} X_{j}=\sum_{j=1}^{10 n}\left(d \mathcal{P}_{j}+W_{j}\right) X_{j}= \\
\sum_{j=1}^{10 n} D \mathcal{P}_{j} X_{j}
\end{array}
$$

where $X_{j}$ is an element of $\boldsymbol{X}^{j}$ and $W_{j}$ is a constant. If the following conditions hold:

$$
D \mathcal{C}_{j}=0 \quad \text { and } \quad d \mathcal{P}_{j}=0
$$

the parameter $X_{j}$ has no effect on the dynamic model and does not belong to the base parameter set. Inertial parameter satisfying (6) are those of the axis near the base side, and there exist rules (Gautier and Khalil, 1988; Gautier and Khalil, 1990) to find those parameters without using (6).

For some other parameters we can write:

$$
D \mathcal{C}_{j}=\alpha_{j 1} D \mathcal{C}_{j 1}+\ldots+\alpha_{j r} D \mathcal{C}_{j r}=\sum_{k=1}^{r} \alpha_{j k} D \mathcal{C}_{j k}
$$

and

$$
d \mathcal{P}_{j}=\alpha_{j 1} d \mathcal{P}_{j 1}+\ldots+\alpha_{j r} d \mathcal{P}_{j r}=\sum_{k=1}^{r} \alpha_{j k} d \mathcal{P}_{j k}
$$

so that the parameter $X_{j}$ is a linear combination of other parameters.

\subsection{Identification Method}

Using the base parameters set we can write the model as a linear regression:

$$
\boldsymbol{\Phi}(\boldsymbol{q}, \dot{\boldsymbol{q}}, \ddot{\boldsymbol{q}}) \boldsymbol{\theta}=\boldsymbol{\tau}
$$

where $\boldsymbol{\theta}=\left[\boldsymbol{\theta}_{B}, \boldsymbol{\theta}_{f}\right]^{\top}$ is the vector of parameters to be estimated, with $\boldsymbol{\theta}_{B}$ the base parameters vector and $\boldsymbol{\theta}_{f}$ the friction parameters vector. $\boldsymbol{\Phi}$ is the linear regressor which include dynamic and friction model of the robot, function of the joint position, velocity and acceleration, while $\tau$ is the command torque.

Different methods exist (Ljung, 1987) to identify $\boldsymbol{\theta}$ in (9); all these methods are valid if the plant is in open loop, but, since the robot operates in closed loop for safety reasons, some of these methods may fail when applied to it. Figure 1 represents a closed-loop system, where $G$ is the system to be identified, $C$ the controller, $H$ models the noise dynamics, $e(t)$ is a white noise disturbance, $r(t)$ is the reference signal, $u(t)$ the torque command and $y(t)$ the measured position of the robot.

The Projection Method (PM) (Forssell and Ljung, 1999; Forssell and Ljung, 2000) has been used here; it is a particular case of joint input-output method that tries to identify the controller from the measurement, with no hypothesis about its structure. PM is a two stage method, as follows:

(1) In the first stage, the sensivity function $S$ is estimated using a non causal FIR (Finite Impulse Response) filter from the equation: 


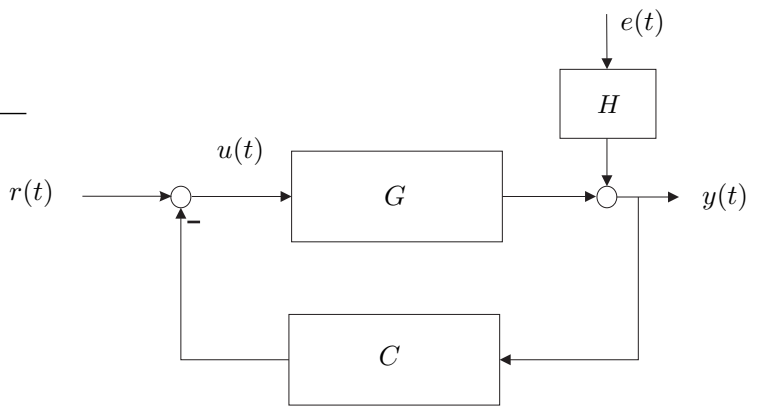

Fig. 1. SISO Closed-loop system.

$$
\begin{aligned}
u(t) & =S(q, \boldsymbol{\theta}) r(t)+H_{2}(q, \boldsymbol{\theta}) e(t) \\
& =\sum_{k=-M_{1}}^{M_{2}} s_{k} r(t-k)+H_{2}(q, \boldsymbol{\theta}) e(t)(10)
\end{aligned}
$$

Using the estimated sensivity $\hat{S}$, an estimate of the torque command is computed as:

$$
\hat{u}(t)=\hat{S}(q, \boldsymbol{\theta}) r(t)=\sum_{k=-M_{1}}^{M_{2}} s_{k} r(t-k)(11)
$$

where $M_{1}$ and $M_{2}$ are chosen to avoid correlation between $\hat{u}(t)$ and $\tilde{u}(t)=u(t)-\hat{u}(t)$; $q^{-k} r(t)=r(t-k)$ is the time-shift operator.

(2) In the second stage, using the estimated command (11), we can write:

$$
y(t)=G(q, \boldsymbol{\theta}) \hat{u}(t)+H_{1}(q, \boldsymbol{\theta}) e(t)
$$

and employ this equation to estimate the system transfer function $G$ with one of the identification methods valid for open-loop systems. $H_{1}$ and $H_{2}$ are suitable functions of $G, C$ and $H$.

This method finds the best projection of the torque command $u(t)$ in the space defined by the reference $r(t)$, obtaining an estimate $\hat{u}(t)$ not influenced by the closed-loop control.

2.2.1. Maximum Likelihood Estimation The second stage of the PM method requires a procedure to estimate the parameters in (12). We use a Maximum Likelihood Estimation algorithm (MLE) (Olsen, 2003) that estimates the value of $\boldsymbol{\theta}$ maximizing the likelihood of the positions $\boldsymbol{q}(t)$ and torque $\boldsymbol{\tau}(t)$ measurements. Since noise on the measurements is always present, it is assumed to be white gaussian with zero mean and variance $\boldsymbol{\sigma}^{2}$. The estimate $\hat{\boldsymbol{\theta}}_{M L}$ of $\boldsymbol{\theta}$ solves the constrained optimization problem:

$$
\min \frac{1}{2} \sum_{i, j=1}^{n}\left({ }^{i} \boldsymbol{x}-{ }^{j} \boldsymbol{\varphi}\right)^{\top} \sigma_{i j}^{-2}\left({ }^{i} \boldsymbol{x}-{ }^{j} \boldsymbol{\varphi}\right)
$$

s. t.

$$
G_{k}\left(\boldsymbol{\theta},{ }^{i} \boldsymbol{\varphi}\right)=0 \quad \forall i, k
$$

where ${ }^{i} \varphi$ is the unknown true value of the $i$ th observation and ${ }^{i} \boldsymbol{x}$ is the measurement corrupted by noise ${ }^{i} \boldsymbol{x}={ }^{i} \boldsymbol{\varphi}+{ }^{i} \boldsymbol{\epsilon}, \forall i=1, \ldots n$, where $n$ is the observation number and $G_{k}$ is the dynamic model for the link $k$.

From (13) the maximum likelihood estimation of $\boldsymbol{\theta}$ is obtained:

$$
\hat{\boldsymbol{\theta}}_{M L}=\arg \min _{\boldsymbol{\theta}} \frac{1}{2} \sum_{i=1}^{n}{ }^{i} \boldsymbol{b}(\boldsymbol{\theta})^{\top}{ }^{i} \boldsymbol{S}_{M L}(\boldsymbol{\theta})^{-2}{ }^{i} \boldsymbol{b}(\boldsymbol{\theta})
$$

where:

$$
\begin{array}{r}
{ }^{i} \boldsymbol{b}(\boldsymbol{\theta})=-\left[\begin{array}{c}
G_{1}\left(\boldsymbol{\theta},{ }^{i} \boldsymbol{x}\right) \\
\vdots \\
G_{6}\left(\boldsymbol{\theta},{ }^{i} \boldsymbol{x}\right)
\end{array}\right] \\
{ }^{i} \boldsymbol{B}(\boldsymbol{\theta})=\left[\begin{array}{c}
\nabla^{{ }^{i} \boldsymbol{x}} G_{1}\left(\boldsymbol{\theta},{ }^{i} \boldsymbol{x}\right) \\
\vdots \\
\nabla_{{ }^{*} \boldsymbol{x}} G_{6}\left(\boldsymbol{\theta},{ }^{i} \boldsymbol{x}\right)
\end{array}\right] \\
{ }^{i} \boldsymbol{S}_{M L}(\boldsymbol{\theta})^{2}={ }^{i} \boldsymbol{B}(\boldsymbol{\theta}){ }^{i} \boldsymbol{\sigma}^{2}{ }^{i} \boldsymbol{B}(\boldsymbol{\theta})^{\top}
\end{array}
$$

Equation (16) gives the covariance matrix of the model parameters estimated with the MLE.

\subsection{Generation of the Robot Trajectories}

An appropriate exciting robot trajectory is necessary to identify $\boldsymbol{\theta}$. We should consider that the observation time is finite and aliasing errors are present; to avoid leakage errors, a periodic excitation trajectory has been planned and repeated measurements collected to average the data and obtain a white gaussian noise error, according to the Central Limit Theorem.

Joint positions and torques are the only data ready for use, since no information about joint velocities and accelerations is available. If the above quantities are analytic functions of the position, then velocity and acceleration can be computed by analytical derivation; for this reason an exciting trajectory given by the following Finite Fourier series has been selected (Swevers et al., 1997):

$$
\begin{gathered}
q_{i}(t)=\sum_{k=1}^{N_{i}}\left\{\frac{a_{i}(k)}{2 \pi f_{0} k} \sin \left(2 \pi f_{0} k t\right)-\right. \\
\left.\frac{b_{i}(k)}{2 \pi f_{0} k} \cos \left(2 \pi f_{0} k t\right)\right\}+q_{i 0} \\
\dot{q}_{i}(t)=\sum_{k=1}^{N_{i}}\left\{a_{i}(k) \cos \left(2 \pi f_{0} k t\right)+\right. \\
\left.b_{i}(k) \sin \left(2 \pi f_{0} k t\right)\right\} \\
\ddot{q}_{i}(t)=\sum_{k=1}^{N_{i}}\left\{\begin{array}{r}
-\left(a_{i}(k) 2 \pi f_{0} k\right) \sin \left(2 \pi f_{0} k t\right)+ \\
\left.\left(b_{i}(k) 2 \pi f_{0} k\right) \cos \left(2 \pi f_{0} k\right)\right\}
\end{array}\right.
\end{gathered}
$$

where $f_{0}$ is the fundamental frequency and $N_{i}$ the number of harmonics. The choice of $f_{0}$ and 
$N_{i}$ determines the bandwidth of the exciting trajectory and we can choose a specific bandwidth to avoid excitation of the elastic modes of the structure. Moreover, the reference signal should be persistently exciting with an order greater or equal to the model order we want to identify.

With this exciting trajectory only the $2 N_{i}+1$ parameters

$$
\boldsymbol{\delta}_{i}=\left[a_{i}(1), \ldots, a_{i}\left(N_{i}\right), b_{i}(1), \ldots, b_{i}\left(N_{i}\right), q_{i 0}\right]^{\top}
$$

$\forall i=1, \ldots, 6$ are to be computed. The vector $\boldsymbol{\delta}_{i}$ shall maximize the information of the observations; to do this (Presse and Gautier, 1993) the Singular Value Decomposition (SVD) of the regressor $\boldsymbol{\Phi}$ was considered and its condition number $\kappa(\boldsymbol{\Phi})$ computed so that the optimal parametrization of the exciting trajectory is given by:

$$
\hat{\boldsymbol{\delta}}_{i}=\arg \min _{\boldsymbol{\delta}_{i}} \kappa(\boldsymbol{\Phi})
$$

subject to given position, velocity and acceleration constrains on each joint and end-effector constrains.

\section{TEST CASE: COMAU SMART-3 S2 ROBOT}

The PM approach was tested on a COMAU Smart-3 S2 robot with $n=6$ revolute joints. A joint-decoupled model of the robot can be used since each joint has a gearbox with an high gear ratio that reduces the interaction among joints. To estimate the dynamic parameters, one joint at a time was moved, while the others were kept static, so that only its inertia and gravity effects are to be taken into account.

\subsection{The Robot Model}

Each joint was modelled by the Lagrange equation (1):

$$
\ddot{q}_{i}(t)=-\frac{h_{i}\left(\boldsymbol{q}, \dot{q}_{i}\right)}{M_{i}}+\frac{\tau_{i}(t)}{M_{i}}
$$

where $M_{i}$ is the equivalent mass value of the $i$-th link, and $h_{i}\left(\boldsymbol{q}, \dot{q}_{i}\right)$ includes gravity and friction effects (2):

$$
h_{i}\left(\boldsymbol{q}, \dot{q}_{i}\right)=g_{i}(\boldsymbol{q})+f_{i}^{f}\left(\dot{q}_{i}\right)
$$

\subsection{Friction Model}

As can be seen in (22), the unknown friction parameters are part of the linear regressor (9). Consequently the friction model has an important role in determining the parametrization of the trajectory (20), but increases the complexity and computing time. To reduce them it is possible to estimate the friction parameters in (2) with specific observations and use them in the generation of the trajectory necessary for parameters identification in (21).
3.2.1. Gravity Compensation Gravity has remarkable effects on the robot behaviour, and that these effects must be compensated. Except for the second and third joint, it is possible to position the robot so to have the other axes parallel to the gravity vector, compensating its effects.

If we model the link $i$ as an inverse pendulum with mass $M$ (mass of the links towards the end effector) on the tip, the gravity model is approximately sinusoidal:

$$
\tau_{g i}=k_{i} \sin \left(q_{i}+q_{i}^{\text {off }}\right)
$$

where $k_{i}$ is the amplitude of the torque gravity and $q_{i}^{\text {off }}$ models the offset position of the center of mass toward the center of the link.

In order to be able to neglect inertia effects and have constant Coriolis, centripetal and friction effects, a slope trajectory is used: in particular two slope trajectories, with the same velocity amplitude but opposite sign are used. The results are reported in Figure 2, where we observe a sinusoidal torque, modelled in (2), and a constant offset due to various effects (centripetal, Coriolis and friction) that depend linearly on the constant velocity of the trajectories. The gravity parameter were estimated (see Table 1), and plotted in Figure 2 (dashed line); the torque was estimated according to (2). Using these parameters we can proceed with the friction identification for axes 2 and 3 .
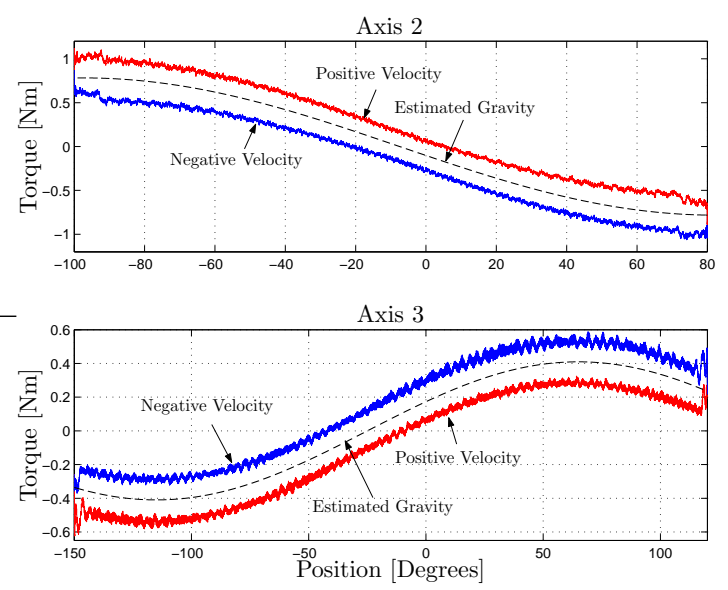

Fig. 2. Measured torque and estimated gravity torque.

3.2.2. Friction Identification Trajectories that enable to recover the information about viscous and Coulomb friction, as modelled in (2), must be generated. A bang-bang acceleration trajectory was used for all links; with this trajectory the inertia has opposite effects, since during acceleration it contrasts the joint motion causing a boost of the torque, and in deceleration it bears the joint motion causing a reduction of the torque. 
For space reasons, only the results of one axis are reported. As expected, plotting the measurement for axis 2 in Figure 3, one can notice an hysteresis due to the link inertia and a bending of the plot due to the gravity effects. Compensating the hysteresis and the gravity, with the parameters from Table 1, we found a value of the torque to be associated only to the friction effects (Coriolis and centripetal effects are neglected in this discussion). The friction parameters are estimated plugging this value of the torque in a Least Square method: the results, reported in Table 2 were used to estimate the friction torque (dashed-line in Figure 3).

\subsection{Trajectory Generation}

The trajectory used in the experiment was based on the method presented in Section 2.3, with an appropriate choice of the bandwidth and of the excitation order. Since the COMAU Smart-3 S2 robot has a resonance frequency between 3 and 20 $\mathrm{Hz}$ for the shoulder joints, and between 5 and 30 $\mathrm{Hz}$ for the wrist joints, the order of the joint model is 2 and the sampling frequency is $f_{s}=1 \mathrm{kHz}$, we choose a fundamental frequency of $f_{0}=0.1$ Hz with $N_{i}=5$ harmonics and a bandwidth of $B_{w}=0.5 \mathrm{~Hz}$, which is considerably less than the minimum resonance frequency of the shoulder joints. With the values of the estimated friction parameters, a trajectory has been generated that allows to solve the constrained optimization problem (20), using the fmincon function of the Matlab Optimization Toolbox; the iteration process was stopped after 2000 iterations. Figure 4 shows the resulting trajectory for link 3 .

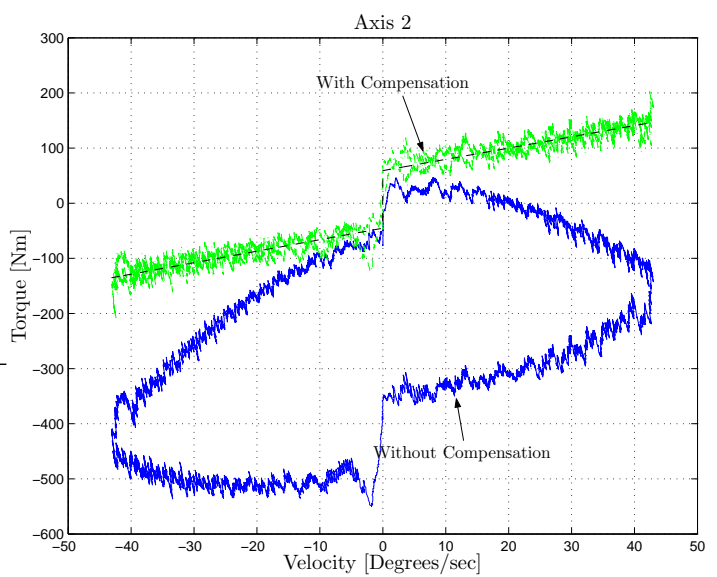

Fig. 3. Measured torque without gravity compensation.

\subsection{Parameters Identification}

Every observation was repeated 25 times to average the data, that were filtered with a 8-th order Chebyshev low pass filter, with cut-off frequency $f_{c}=80 \mathrm{~Hz}$ and resampled at $f_{s}=200 \mathrm{~Hz}$. From the averaged data is possible compute the probability density function (PDF) of the measurement

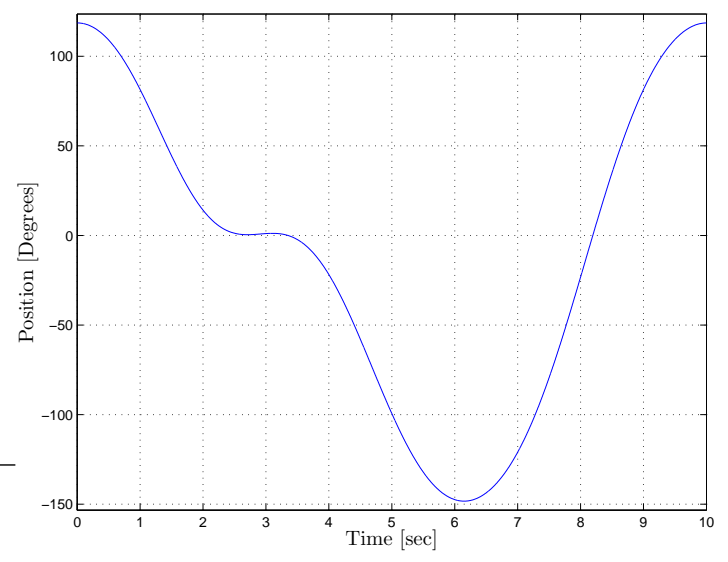

Fig. 4. Generated trajectory for axis 3 .

noise and compare it with a gaussian one (see Figure 5). In Figure 5 one observes that the torque noise is greater than position noise so that we can neglect the position noise and consider only the torque noise.

The measured torque includes friction effects and, to correctly identify robot parameters, one should compensate them using the friction parameters in Table 2, obtaining a value of the torque that depends only on the robot dynamics (see Figure 6 ) and can be used in the identification process.

In the first step of the PM procedure the excitation spectrum was computed using the Fast Fourier Transform (FFT), that has spectrum lines only at $0.1 \mathrm{~Hz}$ and its multiples until $0.5 \mathrm{~Hz}$.

If we compute the actual torque spectrum we find that there are some components over $0.5 \mathrm{~Hz}$, apparently due to the presence of the controller, that gives rise to a correlation between the noise measurements. The best estimate for this torque is obtained filtering the frequencies greater than the bandwidth and considering only the frequencies lower than $0.5 \mathrm{~Hz}$ (see Figure 7); thus a coefficient of correlation between the estimate $\hat{\tau}_{3}$ and the estimation error $\tilde{\tau}_{3}=\tau_{3}-\hat{\tau}_{3}$ was obtained as $\Phi_{\hat{\tau}_{3} \tilde{\tau}_{3}}=2.9238 \mathrm{e}^{-14}$.

In the second step, the robot model parameters were estimated using a MLE torque $\hat{\tau}_{i}$. They are summarized in Table 3; for each link, the following quantities are reported: the parameter symbol with its unit of measure, the mean value and the standard deviation obtained in the identification process and the half amplitude of the $99.9 \%$ confidence interval with $t_{\gamma}$, found using the Student distribution with $(n-1)$ DoF. The analysis of the results shows, as expected, that the first moments for axis 2 and 3 are greater than the others, due to the gravity effects. Axis 2 inertia is the largest, with greatest standard deviation and confidence interval, due to its large variation with respect to robot configuration space. The inertia of the wrist links are lower than those of the shoulder links, as one expects from their smaller size. 


\subsection{Model Validation}

The model was validated comparing the real behaviour of the robot with the one simulated using the estimated parameter values from Table 3. Reference trajectories with trapezoidal velocity shape were used, moving one joint a time. The measurements were filtered by a 8-th order Chebyshev low pass filter. The error between simulated and measured data was computed together with its PDF. The position error was always lower than $0.1^{\circ}$, while the torque error was lower than $0.2 \mathrm{Nm}$. The error PDFs were compared with gaussian ones (see Figure 8) and, apart from the distribution relative to the first link, all others are in good accordance with gaussian distribution; therefore we can conclude that the estimated model is a valid approximation of the true system in the assumed frequency range.

\subsection{Model-Based Controller Design}

A model-based controller was designed for the 6-th joint, on the basis of the estimated model parameters, in order to test the relevance of the identification procedure for control purposes, then implemented on the Linux-RTAI real-time architecture on the PC connected with the robot in our Laboratory, as described in (Alotto et al., 2004).

In Figure 9 the track error is reported, relative to two different controllers, i.e. the original controller used during the identification process (a simple PID-type control) and that based on the identified model. We could see that, after the initial transient, a reduction of the maximum error peak occurs when there is a change of sign in the velocity; furthermore the model-based controller is faster than original.

We are aware that these are only preliminary results and the controller needs still to be tested on the entire structure and tuned to improve its performances.

\section{CONCLUSION}

The aim of this work was to identify the model of an industrial robot and use it in modelbased (computed torque) controller design. A rigid structure was assumed, so its range of validity is limited to low frequencies (approximately under $1 \mathrm{~Hz}$ ). A periodic excitation trajectory with a band-limited frequency content was used, in order to compute joints velocities and accelerations analytically. A serious obstacle for a correct estimation of the model parameters was the presence of the original controller in the feedback loop. Neglecting its presence could lead biased or unreasonable parameters; to avoid this fact the Projection Method was used, that is a valid choice in closed-loop systems.

The identification procedure was applied on a COMAU Smart-3 S2 robot moving one joint at time. The experimental result showed that the presented approach is feasible, and we obtained a model that afterward was successfully used for controller design. This controller was tested on the 1-st and 6-th joint.

To further improve the results, the control validation shall be extended to other links, flexible modes of the structure identified and a more accurate model of the friction torques introduced.

\section{REFERENCES}

Alotto, G., B. Bona and T. Calvelli (2004). Prototyping advanced real-time robotic controllers on linux rtai systems with automatic code generation. In: Proceedings of the Mechatronics and Robotics 2004 International Conference. Vol. 2. Aachen. pp. 65-70.

Bona, B. (2002). Modellistica dei Robot Industriali. Celid. Torino. in Italian.

Forssell, U. and L. Ljung (1999). Closed-loop identification revisited. Automatica 35(7), 12151241.

Forssell, U. and L. Ljung (2000). A projection method for closed-loop identification. IEEE Transactions on Automatic Control 45(11), 2101-2106.

Gautier, M. and W. Khalil (1988). A direct determination of minimum inertial parameter of robot. In: Proceedings of the IEEE International Conference on Robotics and Automation. Philadelphia. pp. 1682-1686.

Gautier, M. and W. Khalil (1990). Direct calculation of minimum set of inertial parameters of serial robots. IEEE Transactions on Robotics and Automation 6(3), 368-373.

Kozlowzki, K. (1998). Modelling and Identification in Robotics. Springer.

Ljung, L. (1987). System Identification: Theory for the User. Prentice-Hall. Englewood Cliffs.

Mayeda, H., K. Yoshida and K. Osuka (1990). Base parameter of manipulator dynamic models. IEEE Transactions on Robotics and Automation 6(3), 312-321.

Olsen, M. M. (2003). Parameter Estimation of Robot Models. PhD thesis. The Maersk McKinney Moller Institute for Production Technology, University of Southern Denmark.

Pham, C. M. and M. Gautier (1991). Essential parameters of robots. In: Proceedings of the 30th Conference on Decision and Control. Bristol. pp. 2769-2774.

Presse, C. and M. Gautier (1993). New criteria of exciting trajectories for robot identification. In: Proceedings of the IEEE International Conference on Robotics and Automation. Vol. 3. pp. 907-912.

Sun, L., H. Ohmori and A. Sano (2000). Direct closed-loop identification approach to unstable plant. In: Proceedings of the $39^{\text {th }}$ IEEE 
Conference on Decision and Control. Vol. 2. Sydney. pp. 1148-1153.

Swevers, J., C. Ganseman, D. B. Tükel, J. De Schutter and H. Van Brussel (1997). Optimal robot excitation and identification. IEEE Transactions on Robotics and Automation 13(5), 730-740.

Welsh, J. S. and G. C. Goodwin (2002). Finite sample properties of indirect non parametric closed-loop identification. IEEE Transactions on Automatic Control 47(8), 1277-1292.

Table 1. Estimated Gravity Parameters

\begin{tabular}{|c|c|c|}
\hline Parameter & Axis 2 & Axis 3 \\
\hline$k$ & $-0,7823 \mathrm{Nm}$ & $0,4097 \mathrm{Nm}$ \\
$q^{\text {off }}$ & $7,5^{\circ}$ & $25^{\circ}$ \\
\hline
\end{tabular}

Table 2. Estimated Friction Parameters

\begin{tabular}{|c|c|c|c|c|}
\hline Parameter & $\beta^{v+}$ & $\beta^{v-}$ & $\beta^{c+}$ & $\beta^{c-}$ \\
\hline \hline Axis 1 & 31,8757 & 26,0000 & 16,1376 & 15,0676 \\
Axis 2 & 36,6197 & 73,0530 & 65,4187 & 45,9598 \\
Axis 3 & 10,6964 & 11,5955 & 13,3809 & 11,3347 \\
Axis 4 & 3,0686 & 3,1806 & 2,0526 & 1,9685 \\
Axis 5 & 3,5672 & 3,2386 & 1,7545 & 1,7161 \\
Axis 6 & 1,8245 & 1,9985 & 2.4545 & 2.4161 \\
\hline
\end{tabular}

Table 3. Estimated Robot Model Parameters

\begin{tabular}{|c||cr|c|c|c|}
\hline Axis & \multicolumn{2}{|c|}{ Parameter } & $\bar{\theta}$ & $\sigma$ & $\frac{t_{\gamma}}{\sqrt{n-1}} \sigma$ \\
\hline \hline 1 & $\Gamma_{1}^{z z}$ & {$\left[\mathrm{kgm}^{2}\right]$} & 28,7822 & 0,3207 & 0,2179 \\
\hline \multirow{3}{*}{2} & $\Gamma_{2}^{z z}$ & {$\left[\mathrm{kgm}^{2}\right]$} & 64,1881 & 1,8838 & 1,2800 \\
& $m x_{2}$ & {$[\mathrm{kgm}]$} & 39,9920 & 0,2252 & 0,1530 \\
& $m y_{2}$ & {$[\mathrm{kgm}]$} & 6,0617 & 0,0539 & 0,0366 \\
\hline \multirow{4}{*}{3} & $\Gamma_{3}^{z z}$ & {$\left[\mathrm{kgm}^{2}\right]$} & 7,4838 & 0,1214 & 0,0825 \\
& $m x_{3}$ & {$\left[\mathrm{kgm}^{2}\right]$} & 2,8006 & 0,0115 & 0,0078 \\
& $m y_{3}$ & {$[\mathrm{kgm}]$} & $-5,6097$ & 0,0147 & 0,0100 \\
\hline \multirow{3}{*}{4} & $\Gamma_{4}^{z z}$ & {$\left[\mathrm{kgm}^{2}\right]$} & 0,2844 & 0,0107 & 0,0072 \\
& $m x_{4}$ & {$[\mathrm{kgm}]$} & $-0,0257$ & 0,0035 & 0,0024 \\
& $m y_{4}$ & {$[\mathrm{kgm}]$} & 0,0063 & 0,0048 & 0,0033 \\
\hline \multirow{3}{*}{5} & $\Gamma_{5}^{z z}$ & {$\left[\mathrm{kgm}^{2}\right]$} & 0,3541 & 0,0099 & 0,0067 \\
& $m x_{5}$ & {$[\mathrm{kgm}]$} & $-0,0518$ & 0,0030 & 0,0020 \\
& $m y_{5}$ & {$[\mathrm{kgm}]$} & 0,1428 & 0,0028 & 0,0019 \\
\hline \multirow{3}{*}{6} & $\Gamma_{6}^{z z}$ & {$\left[\mathrm{kgm}{ }^{2}\right]$} & 0,4601 & 0,0436 & 0,0296 \\
& $m x_{6}$ & {$[\mathrm{kgm}]$} & 0,0313 & 0,0152 & 0,0103 \\
& $m y_{6}$ & {$[\mathrm{kgm}]$} & $-0,0194$ & 0,0169 & 0,0115 \\
\hline
\end{tabular}


Fig. 5. PDF of the measurement noise for axis 3 .

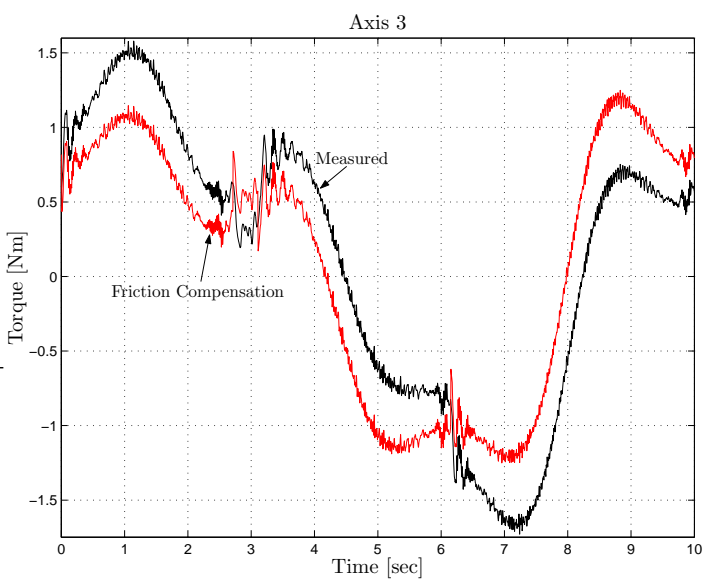

Fig. 6. Measured torque with and without friction compensation.

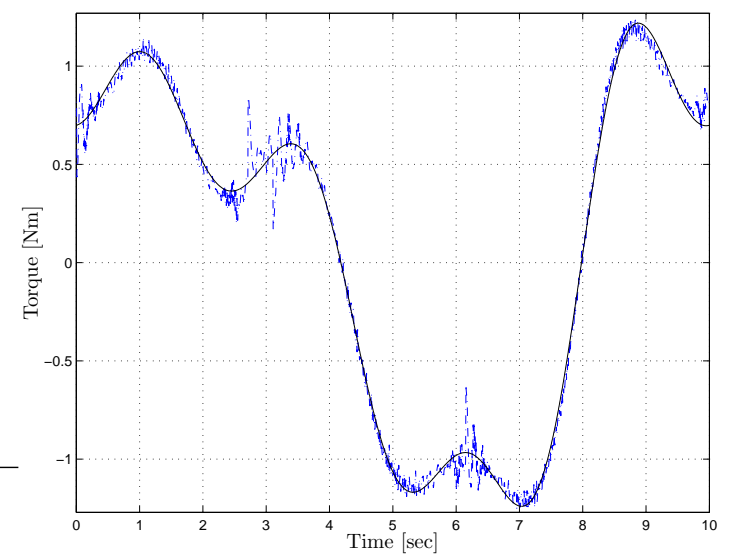

Fig. 7. Axis 3 torque (dash-dotted line: measured, solid line: estimated). 

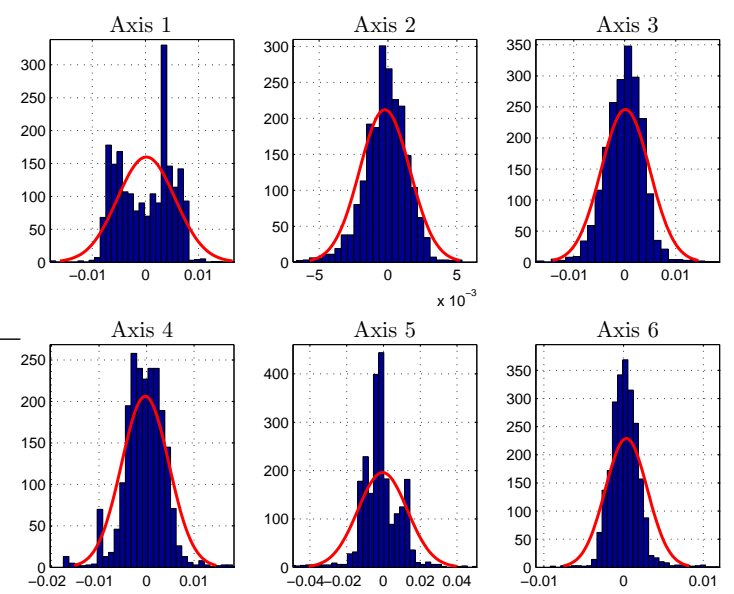

Fig. 8. PDF error of the position error.
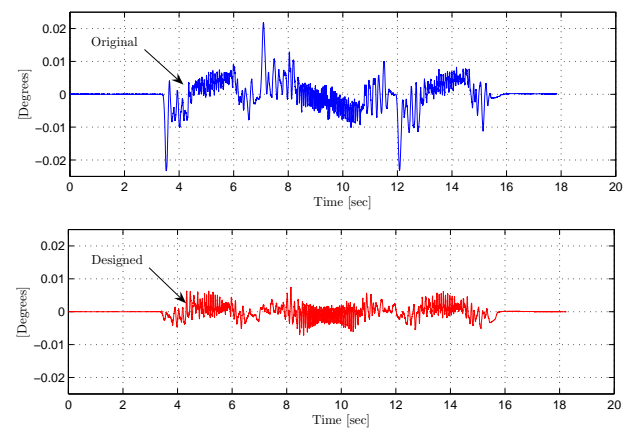

Fig. 9. Controller track error (original and newly designed) for axis 1. 